\title{
Heat and mass transfer in compressible fluid saturated porous media with the boundary element method
}

\author{
R. Jecl ${ }^{1}$, L. Škerget ${ }^{2}$ \& J. Kramer ${ }^{1}$ \\ ${ }^{1}$ Faculty of Civil Engineering, University of Maribor, Slovenia \\ ${ }^{2}$ Faculty of Mechanical Engineering, University of Maribor, Slovenia
}

\begin{abstract}
Most of the studies dealing with transport phenomena in porous media are based on presuming the fluid that saturates the porous domain is incompressible and viscous, where the mass density is a constant quantity, the velocity does not depend on the mass density and pressure is simply a force in the linear momentum equation. However, in numerous natural and engineering systems, density-dependent flow processes play an important role. Besides various applications in the dynamics of pure viscous fluids, such a phenomenon can also be found in subsurface hydrology, geophysics and reservoir mechanics, which are all concerned with problems due to the presence of a permeable solid porous media. In the present work, fully developed boundary element method (BEM) numerical scheme is presented for the simulation of density dependent flow (compressible fluid flow) in porous media with restriction to the subsonic flows. The method is applied to consider heat and mass transfer in a closed porous cavity saturated with compressible fluid, differentially heated under large temperature and concentration gradients. The results in terms of velocity, temperature and species redistribution, as well as the total heat and mass transfer across the cavity, will be presented for different governing parameters.
\end{abstract}

Keywords: porous media, compressible fluid flow, boundary domain integral method, boundary element method, natural convection.

\section{Introduction}

The boundary element method (BEM), which has been established for the viscous incompressible fluid motion in porous media [1], is modified and 
extended to capture the compressible fluid state with restriction to the subsonic flows where the difference in mass density significantly changes the velocity field, but there are no shock waves and no sudden sharp changes in the values of the field functions. Furthermore, the pressure is a thermodynamic quantity, which is temperature and mass density dependent. The flow is modelled by utilizing the Brinkman extended Darcy momentum equation (Brinkman model), which is commonly used when it is important to satisfy the no-slip boundary condition on impermeable surfaces that bound the porous media domain. The governing equations are transformed by using the velocity-vorticity variables formulation and therefore the computation scheme is partitioned into kinematic and kinetic parts.

\section{Mathematical formulation}

The fundamental processes of flow and transport in porous media are presented by the standard continuum approach. The physical properties such as velocity, pressure, temperature and concentration are continuously distributed in space and thus exist for any infinitely small material point. However, in practical problems of course, mass, motion, energy and concentration related quantities cannot be measured and solved at the microscopic level due to the geometric complexity of the real porous domain. Therefore, transformation to the macroscopic level by averaging over representative elementary volume is required. This procedure leads to measurable and solvable quantities for which the continuum approach is then invoked. The basis of density dependent flow and the transport model is stated by the fundamental physical principles of the conservation of mass, momentum, energy and species [2]:

$$
\begin{gathered}
\frac{\partial \phi \rho}{\partial t}+\frac{\partial \rho v_{j}}{\partial x_{j}}=0 \\
\rho\left[\frac{\partial v_{i}}{\partial t}+v_{j} \frac{\partial}{\partial x_{j}}\left(\frac{v_{i}}{\phi}\right)\right]=-\phi \frac{\partial p}{\partial x_{j}}+\phi \rho g_{i}+(\mu+\hat{\mu}) \frac{\partial^{2} v_{j}}{\partial x_{i} \partial x_{j}}-\frac{\mu \phi}{K} v_{j} \\
\frac{\partial}{\partial t}\left[\phi\left(\rho c_{p}\right)_{f}+(1-\phi)\left(\rho c_{p}\right)_{s}\right] T+\frac{\partial}{\partial x_{j}}\left[\phi\left(\rho c_{p}\right)_{f} \frac{v_{j}}{\phi} T\right]=\frac{\partial}{\partial x_{j}} \lambda_{e} \frac{\partial T}{\partial x_{j}} \\
\phi \frac{\partial C}{\partial t}+\frac{\partial v_{j} C}{\partial x_{j}}=\frac{\partial}{\partial x_{j}}\left(D \frac{\partial C}{\partial x_{j}}\right)
\end{gathered}
$$

The parameters used in equations are: $v_{i}$ volume-averaged velocity, $x_{i}$ the ith coordinate, $\phi$ porosity, $t$ time, $\rho$ density, $\mu$ dynamic viscosity, $\partial p / \partial x_{j}$ the pressure gradient, $g_{i}$ gravity, $K$ permeability of porous media, $\hat{\mu}$ coefficient of bulk viscosity, $T$ is temperature, $\lambda_{e}$ the effective thermal conductivity of the 
porous media given as $\lambda_{e}=\phi \lambda_{f}+(1-\phi) \lambda_{s}$, where $\lambda_{f}$ and $\lambda_{s}$ are thermal conductivities for the fluid and solid phases, respectively. Furthermore, $\left(\rho c_{p}\right)_{f}$ and $\left(\rho c_{p}\right)_{s}$ represent heat capacity for the fluid and solid phases, $C$ is concentration and $D$ is used for mass diffusivity. Introducing new variables $\rho^{\prime}=\rho \phi$ and $v_{j}^{\prime}=v_{j} / \phi$, and with the definition of Stokes material derivative of the variable $(\cdot)$ as $D(\cdot) / D t=\partial(\cdot) / \partial t+v_{k} \partial(\cdot) / \partial x_{k}$, the continuity equation can be written as

$$
\frac{\partial v_{j}^{\prime}}{\partial x_{j}}=D^{\prime}=-\frac{1}{\rho^{\prime}} \frac{D \rho^{\prime}}{D t}
$$

where $D^{\prime}$ represent a local expansion rate. According to Stokes hypothesis the second viscous coefficient can be taken as $\hat{\mu}=-2 / 3 \mu$. Because of analytical reasons to develop the velocity-vorticity formulation of governing equations, the momentum eq. (2) is worth writing in its extended form [3, 4]

$$
\begin{gathered}
\rho \frac{D v_{i}^{\prime}}{D t}=-e_{i j k} \frac{\partial \mu \omega_{k}^{\prime}}{\partial x_{j}}+2 e_{i j k} \frac{\partial \mu}{\partial x_{j}} \omega_{k}^{\prime}+2 \frac{\partial \mu}{\partial x_{j}} \frac{\partial v_{i}^{\prime}}{\partial x_{j}}-\frac{2}{3} \frac{\partial \mu}{\partial x_{i}} D^{\prime} \\
+\frac{4}{3} \mu \frac{\partial D^{\prime}}{\partial x_{i}}-\frac{\partial p}{\partial x_{j}}+\rho g_{i}-\frac{\mu \phi}{K} v_{j}^{\prime}
\end{gathered}
$$

with the introduction of the vorticity vector $\omega$, representing the curl of the velocity vector,

$$
\omega_{i}=e_{i j k} \frac{\partial v_{k}}{\partial x_{j}}, \quad \frac{\partial \omega_{j}}{\partial x_{j}}=0
$$

and having in mind that in our case the original vorticity is replaced by the socalled compressible vorticity $\omega^{\prime}, \omega^{\prime}=(1 / \phi) \omega$. It is important to stress that porosity $\phi$ is taken to be constant over individual subdomains, but is changeable in respect to the whole computational domain.

Representing the material properties as a sum of a constant and variable part

$$
\mu=\mu_{0}+\tilde{\mu}, \quad \rho=\rho_{0}+\tilde{\rho}, \quad\left(\rho^{\prime} c_{p}\right)_{f}=c_{0}+\widetilde{c}, \quad \lambda_{e}=\lambda_{e 0}+\tilde{\lambda}, \quad D=D_{0}+\widetilde{D}
$$

then the momentum, energy and species equations (6), (3) and (4) are written as

$$
\frac{D v_{i}^{\prime}}{D t}=-e_{i j k} \frac{\partial \mu_{0} \omega_{k}^{\prime}}{\partial x_{j}}-\frac{1}{\rho_{0}} \frac{\partial p}{\partial x_{j}}+\frac{\rho}{\rho_{0}} g_{i}+\frac{1}{\rho_{0}} f_{i}^{m}-\frac{\mu \phi}{\rho_{0} K} v_{j}^{\prime},
$$


6 Fluid Structure Interaction V

$$
\begin{aligned}
& \frac{D T}{D t}=a \frac{\partial^{2} T}{\partial x_{j} \partial x_{j}}+\frac{S_{T}^{m}}{c_{0}}, \\
& \frac{D C}{D t}=\frac{D_{0}}{\rho_{0}^{\prime}} \frac{\partial^{2} C}{\partial x_{j} \partial x_{j}}+\frac{S_{C}^{m}}{\rho_{0}^{\prime}} .
\end{aligned}
$$

where $a$ is thermal diffusivity. In the pseudo body force term $f_{i}^{m}$, pseudo heat source term $S_{T}^{m}$ and pseudo species source term $S_{C}^{m}$ the effects of variable material properties, are included and given by

$$
\begin{gathered}
f_{i}^{m}=-e_{i j k} \frac{\partial \tilde{\mu} \omega_{k}^{\prime}}{\partial x_{j}}+2 e_{i j k} \frac{\partial \mu}{\partial x_{j}} \omega_{k}^{\prime}+2 \frac{\partial \mu}{\partial x_{j}} \frac{\partial v_{i}^{\prime}}{\partial x_{j}}-\frac{2}{3} \frac{\partial \mu}{\partial x_{i}} D^{\prime}+\frac{4}{3} \mu \frac{\partial D^{\prime}}{\partial x_{i}}-\widetilde{\rho} a_{i}, \\
S_{T}^{m}=\frac{1}{c_{0}}\left(\frac{\partial}{\partial x_{j}} \tilde{\lambda}_{e} \frac{\partial T}{\partial x_{j}}-T \rho^{\prime} \frac{D\left(c_{p}\right)_{f}}{D t}-\frac{\partial\left[(1-\phi)\left(\rho c_{p}\right)_{s}\right] T}{\partial t}-\widetilde{c} \frac{D T}{D t}\right) . \\
S_{C}^{m}=\frac{\partial}{\partial x_{j}}\left(\widetilde{D} \frac{\partial C}{\partial x_{j}}\right)-\widetilde{\rho}^{\prime} \frac{D C}{D t} .
\end{gathered}
$$

\section{Numerical method}

The numerical method chosen for this investigation is the Boundary Domain Integral Method (BDIM) based on the classical Boundary Element Method.

The kinematic is given by velocity vector Poisson's equation

$$
\frac{\partial^{2} v_{i}^{\prime}}{\partial x_{j} \partial x_{j}}+e_{i j k} \frac{\partial \omega_{k}^{\prime}}{\partial x_{j}}-\frac{\partial D^{\prime}}{\partial x_{i}}=0
$$

representing the mass conservation equation (1) and the vorticity definition (7), expressing the compatibility and restriction conditions between velocity, vorticity, and mass density field functions.

The vorticity kinetics is given by the vorticity transport equation obtained as a curl of the momentum equation (9) in the form

$$
\begin{gathered}
\frac{D \omega_{i}^{\prime}}{D t}=v_{0} \frac{\partial^{2} \omega_{i}^{\prime}}{\partial x_{j} \partial x_{j}}+\omega_{j}^{\prime} \frac{\partial v_{i}^{\prime}}{\partial x_{j}}-\omega_{i}^{\prime} D^{\prime}+\frac{1}{\rho_{0}} e_{i j k} \frac{\partial \rho g_{k}}{\partial x_{j}} \\
+\frac{1}{\rho_{0}} e_{i j k} \frac{\partial f_{i}^{m}}{\partial x_{j}}-\frac{\mu \phi}{\rho_{0} K} \omega_{i}^{\prime}
\end{gathered}
$$


The vorticity transport equation (16) is a highly nonlinear partial differential equation due to the products of velocity and vorticity having in mind that the velocity is kinematically dependent on vorticity. Due to the buoyancy force and variable material property terms, acting as additional temperature and pressure dependent vorticity source terms, the vorticity transport equation is coupled to the energy and pressure equations, making the numerical procedure very severe.

In the compressible fluid dynamics the pressure is a thermodynamic quantity that is temperature and mass density dependent. Writing the momentum equation (9) for the pressure gradient we have

$$
\frac{\partial p}{\partial x_{i}}=f_{p i}=-\mu_{0} e_{i j} \frac{\partial \omega^{\prime}}{\partial x_{j}}-\rho_{0} a_{i}+\rho g_{i}+f_{i}^{m}-\frac{\mu \phi}{K} v_{j}^{\prime} .
$$

To derive the pressure equation, depending on known field and material functions, the divergence of equation (17) should be calculated, resulting in the elliptic Poisson pressure equation

$$
\frac{\partial^{2} p}{\partial x_{i} \partial x_{i}}-\frac{\partial f_{p i}}{\partial x_{i}}=0
$$

Equations (15), (16), (10), (11) and (18) represent the leading non-linear set of coupled equations to which the weighted residual technique of the BDIM has to be applied in establishing integral representations corresponding to original differential conservation equations. Each of those equations can be written following the general differential conservation equation where the linear differential operator can be either elliptic or parabolic. The velocity potential equation and a pressure equation are recognised as nonhomogeneous elliptic vector Poisson equations, while the formulations of the integral representation for the vorticity kinetics, heat energy kinetics and species kinetics are based on an elliptic diffusion-convection character of the leading partial differential equations. For the numerical approximate solution of the field functions, namely the velocity, vorticity, pressure and temperature, the integral equations are written in a discretized manner in which the integrals over the boundary $\Gamma$ and domain $\Omega$ are approximated by a sum of the integrals over all boundary elements and over all internal cells. In such a way we obtain the matrix form of the equations, which are solved by coupling kinetic and kinematic equations, considering the corresponding boundary and initial conditions. The integral formulation has been presented in detail previously by Jecl et al. [5], therefore only the resulting matrix form of the equations for kinematics, vorticity kinetics, heat energy kinetics and pressure are presented here. As the computational results of the present work are limited to the two-dimensional case, all the subsequent matrix equations are consequently written for the case of planar geometry only.

$$
[\mathbf{H}]\left\{v_{i}^{\prime}\right\}=e_{i j}\left[\mathbf{H}_{\mathbf{t}}\right]\left\{v_{j}^{\prime}\right\}-e_{i j}\left[\mathbf{D}_{j}\right]\left\{\omega^{\prime}\right\}+\left[\mathbf{D}_{i}\right]\left\{\mathbf{D}^{\prime}\right\},
$$




$$
\begin{aligned}
& {[\mathbf{H}]\left\{\omega^{\prime}\right\}=\frac{1}{v_{0}}[\mathbf{G}]\left\{v_{0} \frac{\partial \omega^{\prime}}{\partial n}-\omega^{\prime} v_{n}^{\prime}-\frac{1}{\rho_{0}} e_{i j} \rho g_{i} n_{j}-\frac{1}{\rho_{0}} e_{i j} f_{i}^{m} n_{j}\right\}} \\
& +\frac{1}{v_{0}}\left[\mathbf{D}_{\mathbf{j}}\right]\left\{\omega^{\prime} \widetilde{v}_{j}^{\prime}+\frac{1}{\rho_{0}} e_{i j} \rho g_{i}+\frac{1}{\rho_{0}} e_{i j} f_{i}^{m}\right\} \\
& -\frac{v \phi}{v_{0} K}[\mathbf{B}]\left\{\omega^{\prime}\right\}+\frac{1}{v_{0} \Delta t}[\mathbf{B}]\left\{\omega_{F-1}^{\prime}\right\} \\
& {[\mathbf{H}]\{T\}=\frac{1}{\lambda_{e 0}}[\mathbf{G}]\left\{\lambda_{e} \frac{\partial T}{\partial n}-2 c_{f} v_{n}^{\prime} T\right\}} \\
& -\frac{1}{\lambda_{e 0}}\left[\mathbf{D}_{\mathbf{j}}\right]\left\{\tilde{\lambda}_{e} \frac{\partial T}{\partial x_{j}}-c_{f} v_{j}^{\prime} T-\widetilde{c}_{f} v_{j}^{\prime} T-\bar{c}_{f} \widetilde{v}_{j}^{\prime} T\right\} \\
& +\frac{1}{\lambda_{e 0}}[\mathbf{B}]\left\{T v_{j}^{\prime} \frac{\partial \widetilde{c}_{f}}{\partial x_{j}}+\widetilde{c}_{f} T D^{\prime}-\widetilde{c}_{f} \frac{\partial T}{\partial t}-(1-\phi)(\rho c)_{s} \frac{\partial T}{\partial t}\right\}+\frac{1}{\Delta t}[\mathbf{B}]\left\{T_{F-1}\right\} \\
& {[\mathbf{H}]\{C\}=\frac{\rho_{0}^{\prime}}{D_{0}}[\mathbf{G}]\left\{\frac{D}{\rho^{\prime}} \frac{\partial C}{\partial n}-C v_{n}^{\prime}\right\}} \\
& +\frac{1}{D_{0}}\left[\mathbf{D}_{\mathbf{j}}\right]\left\{\widetilde{D} \frac{\partial C}{\partial x_{j}}-C v_{j}^{\prime}\right\}-\frac{1}{D_{0}}[\mathbf{B}]\left\{\widetilde{\rho}^{\prime} \frac{\partial C}{\partial t}\right\}+\frac{\rho_{0}^{\prime}}{D_{0}} \frac{1}{\Delta t}[\mathbf{B}]\left\{C_{F-1}\right\} \\
& {[\mathbf{H}]\{p\}=\left[\mathbf{D}_{i}\right]\left\{f_{p i}\right\} .}
\end{aligned}
$$

where kinematic viscosity $v_{0}$ is defined as $v_{0}=\mu_{0} / \rho_{0}$. In all above equations the matrices $[\mathbf{H}],[\mathbf{G}],\left[\mathbf{D}_{\mathbf{j}}\right]$ and $[\mathbf{B}]$ are the influence matrices and they are composed of integrals taken over the individual boundary elements and over the internal cells. In order to improve the economics of the computation, and thus widen the applicability of the proposed numerical algorithm, the subdomain technique is used, where the entire solution domain is partitioned into subdomains to which the same described numerical procedure can be applied. The final system of equations for the entire domain is then obtained by adding the sets of equations for each subdomain considering the compatibility and equilibrium conditions between their interfaces, resulting in a much sparse system matrix suitable to solve with iterative techniques. In our case each quadrilateral internal cell represents one subdomain bounded by four boundary elements. The geometrical singularities are overcome by using 3-node discontinuous quadratic boundary elements combined with 9-node corner continuous internal cells. 


\section{Test example}

The extended numerical algorithm was tested on the problem of natural convection in a porous cavity where the vertical walls are held at different temperatures and the horizontal walls at different concentration values. In literature this phenomena is normally called double diffusive natural convection. The enclosure is filled with porous material, which is homogeneous and isotropic. The saturating density dependent fluid and the solid matrix are both in local thermodynamic equilibrium. The flow is assumed to be steady, laminar, and compressible. Detailed presentation of the geometry and boundary conditions are given in Fig. 1.

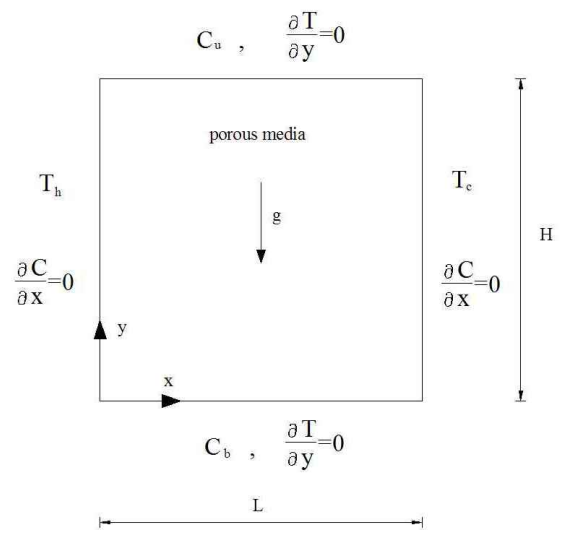

Figure 1: Geometry and boundary conditions for the cavity.

Table 1: $\quad$ Parameters and boundary conditions for the solved problem.

\begin{tabular}{lr}
\hline Dimension of the cavity & $L \times H=0.01 \mathrm{~m} \times 0.01 \mathrm{~m}$ \\
Porosity & $K=10^{-6}, 10^{-7}, 10^{-8} \mathrm{~m}^{2}$ \\
Permeability & $T_{0}=600 \mathrm{~K}$ \\
Reference temperature & $p_{0}=101325 \mathrm{~Pa}$ \\
Reference pressure & $\rho_{0}=0.5884 \mathrm{~kg} / \mathrm{m}^{3}$ \\
Reference density & $\mu_{0}=0.295 \cdot 10^{-4} \mathrm{~Pa} \cdot \mathrm{s}$ \\
Dynamic viscosity & $c_{f}=1004.5 \mathrm{~J} / \mathrm{kg} \mathrm{K}$ \\
Specific heat (fluid phase) & $c_{s}=800 \mathrm{~J} / \mathrm{kg} \mathrm{K}$ \\
Specific heat (solid phase) & $T_{c}=240 \mathrm{~K}$ \\
Temperature of cold wall & $T_{h}=960 \mathrm{~K}$ \\
Temperature of hot wall & $C_{u}=0 \%$ \\
Concentration of upper wall & $C_{b}=1 \%$ \\
Concentration of bottom wall & $D=10^{-6} \mathrm{~m}^{2} / \mathrm{s}$ \\
Coefficient of molecular diffusivity & $R a=10^{3}$ \\
Rayleigh number &
\end{tabular}


The governing parameters for the presented example are given in Table 1. An orthogonal $10 \times 10$ stretched grid in both dimensions was used for computations, where the grid aspect ratio was 6 . The computations were performed for $R a=$ 1000 , where the cold wall is imposed to temperature $T_{c}=240 \mathrm{~K}$ and the hot wall to temperature $T_{h}=960 \mathrm{~K}$, the concentration at the bottom wall is $C_{b}=1 \%$ and at the upper wall $C_{u}=0 \%$. The value of porosity is $\phi=0.1$, the values of permeability are $K=10^{-6}, 10^{-7}, 10^{-8} \mathrm{~m}^{2}$.

The steady state numerical simulation results for the temperature, concentration and velocity fields are presented in Figures 2, 3 and 4.

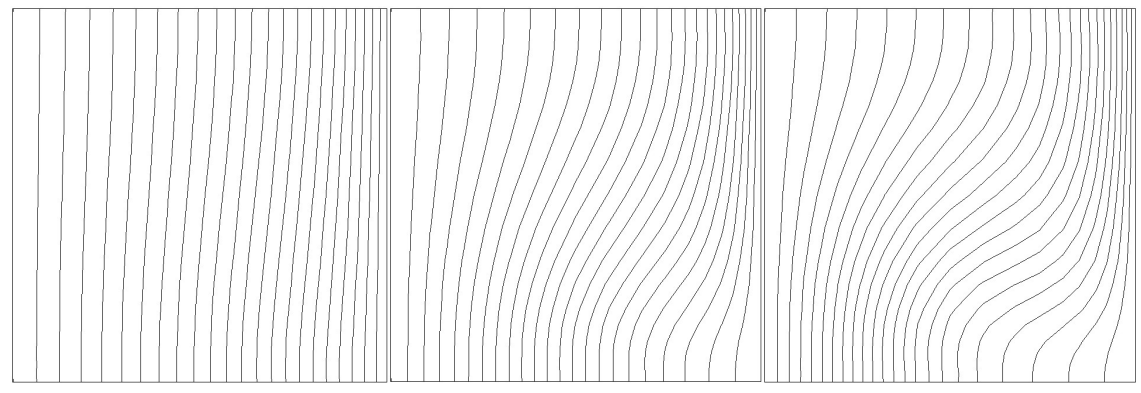

Figure 2: Temperature contours for $R a=10^{3}$, porosity value $\phi=0.1$ and permeability left $K=10^{-6} \mathrm{~m}^{2}$, middle $K=10^{-7} \mathrm{~m}^{2}$, right $K=10^{-8} \mathrm{~m}^{2}$.

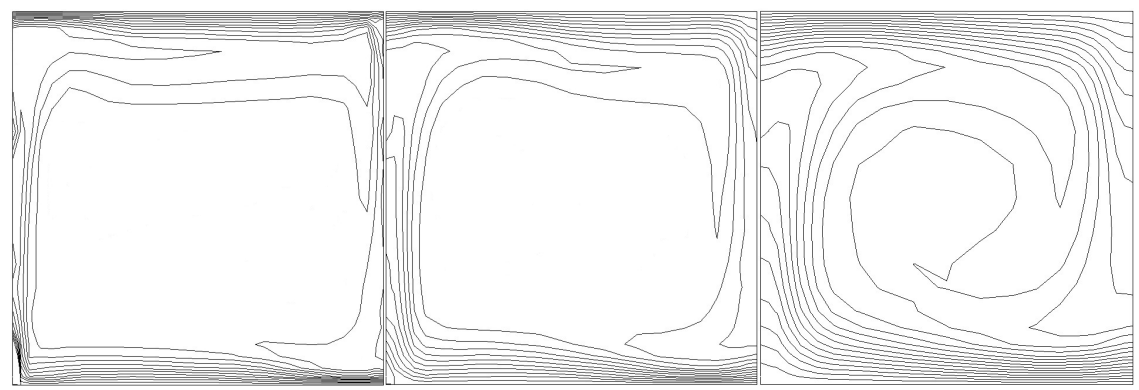

Figure 3: Concentration contours for $R a=10^{3}$, porosity value $\phi=0.1$ and permeability left $K=10^{-6} \mathrm{~m}^{2}$, middle $K=10^{-7} \mathrm{~m}^{2}$, right $K=10^{-8} \mathrm{~m}^{2}$.

The most evident difference in comparison to incompressible fluid flow is the asymmetry of the flow, temperature and concentration fields. As permeability increases the patterns became more and more alike to pure fluid situation.

In the Table 2 the results of overall Nusselt number $N u$ (representing total heat transfer through the cavity) and Sherwood number Sh (representing total mass transfer trough the cavity) for different values of permeability are listed. It is evident that with any decrease in the permeability, the Nusselt and the Sherwood number increases. Identical conclusions can be found in reference [7], while no direct comparison of the results is possible, because of different values of some governing parameters. 


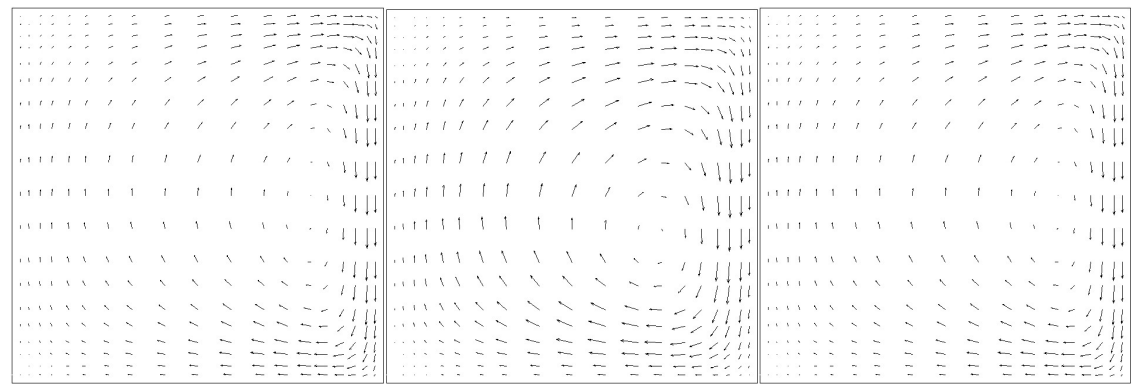

Figure 4: Velocity vectors for $R a=10^{3}$, porosity value $\phi=0.1$ and permeability left $K=10^{-6} \mathrm{~m}^{2}$, middle $K=10^{-7} \mathrm{~m}^{2}$, right $K=10^{-8} \mathrm{~m}^{2}$.

Table 2: $\quad$ Nusselt and Sherwood numbers at different values of $K$ and $\phi=0.1$.

\begin{tabular}{cccc}
\hline & $K=10^{-6}$ & $K=10^{-7}$ & $K=10^{-8}$ \\
\hline $\mathrm{Nu}$ & 1.299 & 1.487 & 1.817 \\
$\mathrm{Sh}$ & 2.033 & 4.891 & 7.825 \\
\hline
\end{tabular}

\section{Conclusion}

The boundary element integral approach for the numerical solution of compressible fluid motion in porous cavity is presented. The derived numerical model is characterized by the decomposition of flow into its kinematics and kinetics, a result of the velocity-vorticity formulation of the modified (porous) Navier-Stokes equation for a compressible fluid. The described numerical algorithm leads to strong coupling between velocity, vorticity and mass density fields, resulting in a stable numerical scheme. The proposed numerical procedure is studied for the case of double diffusive natural convection in square porous cavity. The characteristics of the flow, temperature and concentration fields in the cavity are analysed for different parameters. The results indicated that the BDIM as extended from BEM could be efficiently used also for solving the transport phenomena in porous media saturated with compressible fluid.

\section{References}

[1] Jecl, R., Škerget, L., Boundary element method for natural convection in non-Newtonian fluid saturated square porous cavity, Eng. Anal. Bound. Elem., 27, pp. 963-975, 2003.

[2] Bear, J., Bachmat, Y., Introduction to Modeling of Transport Phenomena in Porous Media, Kluwer Acad. Publ., Dordrecht, Boston \& London, 1991.

[3] Škerget, L., Jecl, R., Compressible fluid dynamics in porous media by the Boundary Element Method (Chapter 6). Emerging Technologies and Techniques in Porous Media, eds.: D.B. Ingham, A. Bejan, E. Mamut, I. Pop, Kluwer Acad. Publ., Dordrecht, Boston \& London, 93-117, 2004. 
[4] Škerget, L., Samec, N., BEM for the two-dimensional plane compressible fluid dynamics, Eng. Anal. Bound. Elem., 29, pp. 1-17, 2005.

[5] Jecl, R., Škerget, L., Kramer, J., Numerical solution of compressible fluid flow in porous media with boundary element method, Proc. of the Int. Conf. Fluid structure interaction and moving boundary problems $I V$, eds. Chakrabarti, S. K., Brebbia, C. A., WIT Transactions on the built environment, Vol. 92, Southampton: WIT Press, pp 143-151, 2007.

[6] Diersch H.-J.G., Kolditz O., Variable-density flow and transport in porous media: approaches and challenges, Advances in Water Resources, 25, pp. 899-944, 2002.

[7] Marpu D.R., Satyamurty V.V., Influence of variable fluid density on free convection in rectangular porous media, Journal of Energy Resources Technology, 111, pp. 214-220, 1989. 